University of Texas at El Paso

ScholarWorks@UTEP

\title{
8-2019
}

\section{Towards a Theoretical Explanation of How Pavement Condition Index Deteriorates over Time}

\author{
Edgar Daniel Rodriguez Velasquez \\ The University of Texas at El Paso, edrodriguezvelasquez@miners.utep.edu \\ Carlos M. Chang Albitres \\ The University of Texas at El Paso, cchangalbitres2@utep.edu \\ Vladik Kreinovich \\ The University of Texas at El Paso, vladik@utep.edu
}

Follow this and additional works at: https://scholarworks.utep.edu/cs_techrep

Part of the Applied Mathematics Commons, and the Construction Engineering and Management

Commons

Comments:

Technical Report: UTEP-CS-19-86

\section{Recommended Citation}

Rodriguez Velasquez, Edgar Daniel; Chang Albitres, Carlos M.; and Kreinovich, Vladik, "Towards a Theoretical Explanation of How Pavement Condition Index Deteriorates over Time" (2019). Departmental Technical Reports (CS). 1362.

https://scholarworks.utep.edu/cs_techrep/1362

This Article is brought to you for free and open access by the Computer Science at ScholarWorks@UTEP. It has been accepted for inclusion in Departmental Technical Reports (CS) by an authorized administrator of ScholarWorks@UTEP. For more information, please contact Iweber@utep.edu. 


\title{
Towards a Theoretical Explanation of How Pavement Condition Index Deteriorates over Time
}

\author{
Edgar Daniel Rodriguez Velasquez ${ }^{1,2}$, Carlos M. Chang Albitres², \\ and Vladik Kreinovich ${ }^{3}$ \\ ${ }^{1}$ Department of Civil Engineering \\ Universidad de Piura in Peru (UDEP) \\ Av. Ramón Mugica 131, Piura, Peru, edgar.rodriguez@udep.pe \\ ${ }^{2}$ Department of Civil Engineering \\ ${ }^{3}$ Department of Computer Science \\ University of Texas at El Paso, 500 W. University \\ El Paso, TX 79968, USA \\ edrodriguezvelasquez@miners.utep.edu, cchangalbitres2@utep.edu, \\ vladik@utep.edu
}

\begin{abstract}
To predict how the Pavement Condition Index will change over time, practitioners use a complex empirical formula derived in the 1980s. In this paper, we provide a possible theoretical explanation for this formula, an explanation based on general ideas of invariance. In general, the existence of a theoretical explanation makes a formula more reliable; thus, we hope that our explanation will make predictions of road quality more reliable.
\end{abstract}

\section{Formulation of the Problem}

The quality of a road pavement is described by a Pavement Condition Index (PCI) that takes into account all possible pavement imperfections [2]. The perfect condition of the road corresponds to $\mathrm{PCI}=100$, and the worst possible condition corresponds to $\mathrm{PCI}=0$.

As the pavement ages, its quality deteriorates. To predict this deterioration, practitioners use an empirical formula developed in [5]:

$$
\mathrm{PCI}=100-\frac{R}{(\ln (\alpha)-\ln (t))^{1 / \beta}},
$$

where $t$ is the pavement's age, and $R, \alpha$ are corresponding parameters. 
In this paper, we propose a possible theoretical explanation for this empirical formula, explanation based on the general notions of invariance. In general, when a formula has a theoretical explanation, it increases the users' confidence in using this formula; this was our main motivation for providing such an explanation.

\section{General Invariances}

Main natural transformations. In order to describe our explanation, let us recall the basic ideas of invariance. Many of these ideas come from the fact that in data processing, we use numerical values of physical properties. In general, for the same physical quantity, we get different numerical values depending on what measuring unit we use and what starting point we use as a 0 value. For example, if we replace meters with centimeters, all numerical values get multiplied by 100 . In general, if we replace the original measuring unit with a new unit which is $\lambda$ times smaller, then each original numerical value $x$ is replaced by the new numerical value $\lambda \cdot x$. This transformation is known as scaling.

Similarly, if for measuring temperature, we select a new starting point which is 32 degrees below the original zero, then this number 32 is added to all the numerical values. In general, if we choose a new starting point which precedes the original one by $x_{0}$ units, then each original numerical value $x$ is replaced by the new numerical values $x+x_{0}$. This transformation is known as shift.

Notion of invariance. The physics does not change if we simply change the measuring unit and/or change the starting point. Thus, it makes sense to require that the physical properties should not change if we apply the corresponding natural transformations: scaling or shift; see, e.g., $[3,6]$. In particular, it makes sense that the relations $y=f(x)$ between physical quantities be thus invariant.

Of course, if we change the measuring unit for $x$, then we may need to change the measuring unit for $y$. For example, the formula $y=x^{2}$ for the area $y$ of the square of linear size $x$ does not depend on the units, but if we change meters to centimeters, we also need to correspondingly change square meters to square centimeters. So, the proper definition of invariance is that for each natural transformation $x \rightarrow X$ there exists an appropriate transformation $y \rightarrow Y$ such that if, in the original scale, we had $y=f(x)$, then in the new scale, we will have $Y=f(X)$.

Invariant dependencies: towards a general description. There are two possible types of natural transformations for $x$ : scaling and shift. Similarly, there are two possible types of natural transformation of $y$. Depending on which class of natural transformation we choose for $x$ and for $y$, we will thus get $2 \cdot 2=4$ possible cases. Let us describe these four cases explicitly, and let us describe, for each of the cases, what are the corresponding invariant functions.

Case of $x$-scaling and $y$-scaling. In this case, for each $\lambda>0$, there exists a value $\mu>0$ such that if we have $y=f(x)$, then for $X=\lambda \cdot x$ and $Y=\mu \cdot y$, we will have $Y=f(X)$. Substituting the expressions for $Y$ and $X$ into this 
formula, and explicitly taking into account that $\mu$ depends on $\lambda$, we get the following equation:

$$
\mu(\lambda) \cdot f(x)=f(\lambda \cdot x) .
$$

All continuous (and even discontinuous but measurable) solutions to this equations are known (see, e.g., [1]): they all have the form

$$
f(x)=A \cdot x^{b}
$$

for some $A$ and $b$.

Comment. The proof for general measurable functions is somewhat complex, but in the natural case of differentiable dependence $f(x)$, the proof is easy. Namely, from the equation (2), we conclude that $\mu(\lambda)=\frac{f(\lambda \cdot x)}{f(x)}$. Since the right-hand side of this equality is differentiable, the left-hand side $\mu(\lambda)$ is differentiable too. Thus, we can differentiate both sides by $\lambda$ and take $\lambda=1$. As a result, we get $\mu_{0} \cdot f(x)=x \cdot d f(x) d x$, where $b \stackrel{\text { def }}{=} \mu^{\prime}(1)$. We can separate the variables $x$ and $f$ by moving all the terms containing $x$ to one side and all the terms containing $f$ to the other side; then, we get $b \cdot \frac{d x}{x}=\frac{d f}{f}$. Integrating both sides, we get $\ln (f)=b \cdot \ln (x)+C$, thus indeed $f=\exp (\ln (f))=A \cdot x^{b}$, where $A=\exp (C)$.

Case of $x$-scaling and $y$-shift. In this case, for each $\lambda>0$, there exists a value $y_{0}(\lambda)$ such that if $y=f(x)$, then $Y=f(X)$ for $Y=y+y_{0}$ and $X=\lambda \cdot x$. Thus, we get

$$
f(x)+y_{0}(\lambda)=f(\lambda \cdot x) .
$$

If the function $f(x)$ is differentiable, then the difference $y_{0}(\lambda)=f(\lambda \cdot x)-f(x)$ is also differentiable. Differentiating both sides of the equation (4) with respect to $\lambda$ and taking $\lambda=1$, we get $b=x \cdot \frac{d f(x)}{d x}$, where $b \stackrel{\text { def }}{=} y_{0}^{\prime}(1)$. Separating the variables, we get $d f=b \cdot \frac{d x}{x}$, and after integration, we get

$$
f(x)=A+b \cdot \ln (x)
$$

for some constant $A$.

Comment. Similarly to the previous case, the same formula (5) holds if we only assume that $f(x)$ is measurable [1].

Case of $x$-shift and $y$-scaling. In this case, for each $x_{0}$, there exists a value $\lambda\left(x_{0}\right)$ such that if $y=f(x)$, then $Y=f(X)$ for $Y=\lambda \cdot y$ and $X=x+x_{0}$. Thus, we get

$$
\lambda\left(x_{0}\right) \cdot f(x)=f\left(x+x_{0}\right) .
$$

If the function $f(x)$ is differentiable, then the ratio $\lambda\left(x_{0}\right)=\frac{f\left(x+x_{0}\right)}{f(x)}$ is also differentiable. Differentiating both sides of the equation (6) with respect 
to $x_{0}$ and taking $x_{0}=0$, we get $b \cdot f(x)=\frac{d f(x)}{d x}$, where $b \stackrel{\text { def }}{=} \lambda^{\prime}(0)$. Separating the variables, we get $\frac{d f}{f}=b \cdot d x$, and after integration, we get $\ln (f)=b \cdot x+C$ hence

$$
f(x)=A \cdot \exp (b \cdot x)
$$

where $A=\exp (C)$.

Comment. Similarly to the previous two cases, the same formula (7) holds if we only assume that $f(x)$ is measurable [1].

Case of $x$-shift and $y$-shift. In this case, for each $x_{0}$, there exists a value $y_{0}\left(x_{0}\right)$ such that if $y=f(x)$, then $Y=f(X)$ for $Y=y+y_{0}$ and $X=x+x_{0}$. Thus, we get

$$
f(x)+y_{0}\left(x_{0}\right)=f\left(x+x_{0}\right) .
$$

If the function $f(x)$ is differentiable, then the difference $y_{0}\left(x_{0}\right)=f\left(x+x_{0}\right)-$ $f(x)$ is also differentiable. Differentiating both sides of the equation (8) with respect to $x_{0}$ and taking $x_{0}=0$, we get $b=\frac{d f(x)}{d x}$, where $b \stackrel{\text { def }}{=} y_{0}^{\prime}(0)$. Thus,

$$
f(x)=A+b \cdot \ln (x)
$$

for some constant $A$.

Comment. In this case too, the same formula (9) holds if we only assume that $f(x)$ is measurable [1].

\section{First Attempt: Let Us Directly Apply Invari- ance Ideas to Our Problem}

PCI and age: scale-invariance or shift-invariance? We are analyzing how PCI depends on the pavement's age $t$. To apply invariances to our dependence, we need first to analyze which invariances are reasonable for the corresponding variables - PCI and age.

For age, the answer is straightforward: there is a clear starting point for measuring age, namely, the moment when the road was built. On the other hand, there is no fixed measuring unit: we can measure age in years or in months or - for good roads - in decades. Thus, for age:

- shift-invariance - corresponding to the possibility of changing the starting point - makes no physical sense, while

- scale-invariance - corresponding to the possibility of changing the measuring unit - makes perfect sense.

For PCI, the situation is similar. Namely, there is a very clear starting point - the point corresponding to the newly built practically perfect road, when PCI 
$=100$. From this viewpoint, for PCI, shifts do not make much physical sense. If we select 100 as the starting point (i.e., as 0), then instead of the original numerical values PCI, we get shifted values PCI -100 .

A minor problem with these shifted values is that they are all negative, while it is more convenient to use positive numbers. Thus, we change the sign and consider the difference 100 - PCI.

On the other hand, the selection of point 0 is rather subjective. What is marked as 0 in a developed country that can afford to invest money into road repairs may be a passable road in a poor country, where most of the roads are, from the viewpoint of US standards, very bad; see, e.g., [4]. So, for PCI (or, to be more precise, for $100-\mathrm{PCI}$ ), it probably makes sense to use scaling.

Let us directly apply the invariance ideas. In view of the above analysis, we should be looking for a dependence of $y=100-$ PCI on $x=t$ which is invariant with respect to $x$-scaling and $y$-scaling. As we have discussed in the previous section, this requirement leads to $y=A \cdot x^{b}$, i.e., to $100-\mathrm{PCI}=A \cdot t^{b}$ and $\mathrm{PCI}=100-A \cdot t^{b}$.

This formula may be reasonable from the purely mathematical viewpoint, but in practice, it is a very crude description of what we actually observe. Thus, the direct application of invariance ideas does not lead to good results.

\section{Let Us Now Apply Invariance Ideas Indirectly}

Idea. Since we cannot apply the invariance requirements directly - to describe the dependence of $y=100-$ PCI on $x=t$, a natural idea is to apply these requirements indirectly. Namely, we assume that there is some auxiliary intermediate variable $z$ such that $y$ depends on $z, z$ depends on $x$, and both these $y$-on- $z$ and $z$-on- $x$ dependencies are, in some reasonable sense, invariant.

Options. We know that for $x$ and for $y$, only scaling makes sense. However, for the auxiliary variable $z$, in principle, both shifts and scalings may be physically reasonable. Depending on which of the two types of transformations we use for $z$ when describing $y$-on- $z$ and $z$-on- $x$ dependencies, we get four possible options:

- for both $y$-on- $z$ and $z$-on- $x$ dependencies, we use $z$-shift;

- for both $y$-on- $z$ and $z$-on- $x$ dependencies, we use $z$-scaling;

- for $y$-on- $z$ dependence, we use $z$-shift, while for $z$-on- $x$ dependence, we use $z$-scaling;

- for $y$-on- $z$ dependence, we use $z$-scaling, while for $z$-on- $x$ dependence, we use $z$-shift.

Let us consider these four cases one by one.

Case when for both $y$-on- $z$ and $z$-on- $x$ dependencies, we use $z$-shift. In this case, in accordance to the results presented in Section 2, we have $z=$ 
$A+b \cdot(x)$ and $y=A_{1} \cdot\left(b_{1} \cdot z\right)$. Substituting the expression for $z$ into the formula for $y$, we get

$$
y=A_{1} \cdot \exp (A+b \cdot \ln (x))=\left(A_{1} \cdot \exp (A)\right) \cdot\left(\exp (\ln (x))^{b}=A_{2} \cdot x^{b},\right.
$$

where $A_{2} \stackrel{\text { def }}{=} A_{1} \cdot \exp (A)$. This is exactly the formula coming from the direct application of invariance requirements, and we already know that this formula is not very adequate for describing the experimental data.

Case when for both $y$-on- $z$ and $z$-on- $x$ dependencies, we use $z$-scaling. In this case, we have $z=A \cdot z^{b}$ and $y=A_{1} \cdot z^{b_{1}}$. Thus, here,

$$
z=A_{1} \cdot\left(A \cdot x^{b}\right)^{b_{1}}=A_{2} \cdot x^{b_{2}},
$$

where $A_{2} \stackrel{\text { def }}{=} A_{1} \cdot A^{\alpha_{1}}$ and $b_{2} \stackrel{\text { def }}{=} b \cdot b_{1}$. Thus, in this case, we also get the same formula as for the direct application of invariance.

Case when for $y$-on- $z$ dependence, we use $z$-shift, while for $z$-on- $x$ dependence, we use $z$-scaling. Here, $z=A \cdot x^{b}$ and $y=A_{1} \cdot \exp \left(b_{1} \cdot y\right)$, thus $y=A_{1} \cdot \exp \left(b_{1} \cdot A \cdot x^{b}\right)$, i.e., $y=A_{1} \cdot \exp \left(b_{2} \cdot x^{b}\right)$, where $b_{2} \stackrel{\text { def }}{=} b_{1} \cdot A$. So, for $\mathrm{PCI}=100-y$ and $x=t$, we get the dependence

$$
\text { PCI }=100-A_{1} \cdot \exp \left(b_{2} \cdot t^{b}\right) .
$$

Interestingly, this is one of the formula that was tested in [5] and which turned out to work not so well as the formula that was selected.

Case when for $y$-on- $z$ dependence, we use $z$-scaling, while for $z$-on- $x$ dependence, we use $z$-shift. In this case, $z=A+b \cdot \ln (x)$ and $y=A_{1} \cdot z^{b}$, thus $y=A_{1} \cdot(A+b \cdot \ln (x))^{b_{1}}$. So, for PCI $=100-y$ and $x=t$, we get

$$
\mathrm{PCI}=100-A_{1} \cdot(A+b \cdot \ln (x))^{b_{1}} .
$$

Let us show that this is indeed the desired formula (1).

Indeed, here,

$$
A+b \cdot \ln (x)=(-b) \cdot\left(\left(-\frac{A}{b}\right)-\ln (x)\right) .
$$

For $\alpha \stackrel{\text { def }}{=} \exp \left(-\frac{A}{b}\right)$, we have $\ln (\alpha)=-\frac{A}{b}$, so the formula (12) takes the form $A+b \cdot \ln (x)=(-b) \cdot(\ln (\alpha)-\ln (t))$. Thus, the formula (11) takes the form

$$
\mathrm{PCI}=100-A_{1} \cdot(-b)^{b_{1}} \cdot(\ln (\alpha)-\ln (t))^{b_{1}},
$$

i.e., the desired form (1) with $R=A_{1} \cdot(-b)^{b_{1}}$ and $\beta=-\frac{1}{b_{1}}$.

Conclusion. We indeed derived the empirical formula (1) for the decrease of PCI over time from the general invariance requirements. To be more precise, from the invariance requirements, we can derive two possible formulas: 
- the desired formula (1) - which is in good accordance with the empirical data, and

- the alternative formula (10) - which is not a good fit for empirical data.

Since in general, the existence of a theoretical explanation makes a formula more reliable, we hope that our explanation will make predictions of road quality more reliable.

\section{Acknowledgments}

This work was partially supported by the Universidad de Piura in Peru (UDEP) and by the US National Science Foundation via grants 1623190 (A Model of Change for Preparing a New Generation for Professional Practice in Computer Science) and HRD-1242122 (Cyber-ShARE Center of Excellence).

\section{References}

[1] J. Aczel and J. Dhombres, Functional Equations in Several Variables, Cambridge University Press, Cambridge, UK, 1989.

[2] American Society for Testing and Materials (ASTM), Standard Test Method for Measuring the Longitudal Profile of Traveled Surfaces with an Accelerometer Established Inertial Profiling Reference, ASTM Standard E950/E950M09, 2018.

[3] R. Feynman, R. Leighton, and M. Sands, The Feynman Lectures on Physics, Addison Wesley, Boston, Massachusetts, 2005.

[4] E. Greenbaum, Emerald Labyrinth: A Scientist's Adventures in the Jungles of the Congo, ForeEdge, Lebanon, New Hampshire, 2018.

[5] Metropolitan Transportation Commission (MTC), Technical Appendices describing the Development and Operation of the Bay Area Pavement Management System (PMS), prepared by Roger E. Smith, 1987.

[6] K. S. Thorne and R. D. Blandford, Modern Classical Physics: Optics, fluids, Plasmas, Elasticity, Relativity, and Statistical Physics, Princeton University Press, Princeton, New Jersey, 2017. 